\title{
MIRACLE - A Joint DLR/ONERA Effort on Harmonization and Development of Industrial and Research Aerodynamic Computational Environment
}

\author{
L. Cambier ${ }^{a}$, N. Kroll ${ }^{b}$ \\ ${ }^{a}$ ONERA, CFD and Aeroacoustics Department, 29 Avenue de la Division Leclerc, 92320 Châtillon, France \\ ${ }^{b}$ DLR, Institute for Aerodynamics and Flow Technology, Lilienthalplatz 7, 38108 Braunschweig, Germany
}

\begin{abstract}
The main objective of the common DLR/ONERA project MIRACLE is to harmonize the CFD activities and strategies of DLR and ONERA in order to better fulfil the future requirements of transport aircraft industry in Europe. The paper presents the most significant achievements of the co-operative activities, which have been focused on the following objectives: collaborative enhancement and industrialization of the structured and unstructured-hybrid CFD solvers elsA (ONERA) and TAU (DLR); validation of both flow solvers elsA and TAU for industrial applications; identification of an appropriate software architecture for next generation CFD tools.

The joint effort of DLR and ONERA on CFD development has accelerated the enhancement of numerical methods for aerodynamic predictions within conventional and unconventional, environmentally friendly aircraft designs. Both, flow solvers elsA and TAU are routinely used in European aircraft industry. The MIRACLE effort is an important step on the road map to meet the future ambitious goals of aircraft research and industry.
\end{abstract}

Keywords: Computational Fluid Dynamics, structured solver, hybrid-unstructured solver, complex configurations, CFD/CSM coupling, software components

\section{Introduction}

CFD has become a mature technology taking a key role at all stages of aircraft design processes. The developments carried out by both DLR and ONERA have had deep impact on the growth of the routine use of CFD tools in European industry. However, future demands of aircraft industry with respect to more environmentally friendly, safer and economical aircraft require further increase in simulation capabilities.

The main objective of the three-year common DLR/ONERA project MIRACLE, launched in 2004, is to harmonize the CFD activities and strategies of DLR and ONERA in order to better fulfil the future requirements of transport aircraft industry in Europe. In connection with the background of the rationalization process of Airbus in the field of CFD tools, the aim of the MIRACLE project is to provide an aerodynamic toolset consisting of a structured multi-block and an unstructured-hybrid mesh based Navier-Stokes solver. Activities have been focused on the following objectives:

- Collaborative enhancement and industrialization of the structured and unstructured-hybrid CFD solvers elsA (ONERA) and TAU (DLR) in order to meet the short- and mid-term requirements of aircraft industry;

- Validation of both flow solvers elsA and TAU for industrial applications and demonstration of enhanced efficiency, accuracy, robustness, and flexibility of the improved methods;

- Identification of an appropriate software architecture for next generation CFD tools for long-term requirements of industry.

We recall that a co-operation on CFD for helicopters had been previously defined between DLR and ONERA (see [1] in this ODAS symposium). MIRACLE project activities have taken advantage of the initial mutual knowledge of the two CFD teams.

The paper reports on status and recent developments of both CFD codes elsA and TAU. It contains simulations demonstrating the capabilities of the aerodynamic computational environment available at DLR and ONERA. It also describes the software architecture work. The conclusion is focused on the most significant achievements and on the perspectives of the DLR/ONERA co-operation on CFD.

\section{Structured multi-block RANS code elsA}

\subsection{General description}

As described in [2], [3], elsA is a multi-application CFD simulation platform dealing with internal and external aerodynamics from the low subsonic to the high supersonic flow regime. The compressible 3-D Reynolds averaged Navier-Stokes equations for arbitrary moving bodies are solved by a cell centered finite-volume method with second order upwind or central space discretization with scalar or matrix artificial dissipation on multi-block meshes. A high flexibility in the multi-block approach is achieved in elsA through patched grid, Hierarchical Mesh Refinement and Chimera [4] techniques. The discrete equations are integrated either by multistage Runge-Kutta schemes with implicit residual smoothing, or, which in general leads to a better efficiency, by backward Euler integration with implicit LU schemes. For time accurate computations, the implicit dual time stepping method or the Gear integration scheme are employed. Preconditioning is used for low speed flow simulations. A large variety of turbulence models are 
available, ranging from eddy viscosity to full differential Reynolds stress models, and including options for DES (see [5] in this symposium) and LES. Various transition models are also available for complex geometry configurations (see [6] in this symposium).

elsA software package includes an important software module dealing with aeroelasticity which is described below. Also, a module dealing with calculation of gradients by linearized equation or by adjoint solver techniques is very useful for aerodynamic optimization (see [7], [8] in this symposium).

The development of elsA is based on Object-Oriented design and implementation since the beginning in 1997. els $A$ is available on a wide range of platforms, including vector and scalar machines. els $A$ also includes a load balancing tool with block-splitting capability, necessary for efficiency in parallel.

Let us present now two examples of recent progress in els $A$ for transport aircraft flow simulations: first example is Differential Reynolds Stress turbulence modelling; second example is aeroelasticity.

\subsection{Turbulence and transition}

Co-operation on turbulence modelling in MIRACLE project has focused on Spalart-Allmaras, $(k, \omega)$ and Differential Reynolds Stress Models. A detailed comparison between the variants implemented in DLR and ONERA software and an exchange of information on the results obtained with these variants have been performed. In particular, in the European project FLOMANIA, DLR and ONERA had developed respectively in the DLR multi-block structured FLOWer code and in elsA, a Differential Reynolds Stress Model based on the Speziale-Sarkar-Gatski model and on the $\varepsilon$-equation as the length scale equation. The analysis of the results obtained by DLR [9], [10] when replacing the $\varepsilon$-equation by the $\omega$ equation has led the ONERA/DMAE Department (Aerodynamics and Energetics Modeling) to consider also in els $A$ the $\omega$-equation of Wilcox or Menter as the length-scale equation associated with the Differential Reynolds Stress Model.

The improvement of the prediction due to the Differential Reynolds Stress Model (here based on the $\omega$-equation of Wilcox) compared with the Spalart-Allmaras model is shown (Fig. 1) on a 2-D high lift configuration of the EUROLIFT II project (Mach number equal to 0.22 , angle of attack equal to $24.4^{\circ}$, Reynolds number equal to 4 million). The calculation is done in a multi-block mesh (87000 points) providing with wall reduced coordinate $\mathrm{y}+$ values less or equal than 1 . The minimum pressure coefficient on the flap, as well as the pressure evolution at the suction side of the flap, are better predicted by the DRSM model. Note that satisfactory results have also been obtained in 3-D with the DRSM functionality of $e l s A$ on a wing/fuselage configuration of the EUROLIFT II project (mesh of 5.5 million points).

DLR and ONERA transition specialists have also exchanged in MIRACLE project detailed information on the respective approaches and prospects. Recent developments in transition in $e l s A$ are presented in this symposium [6].
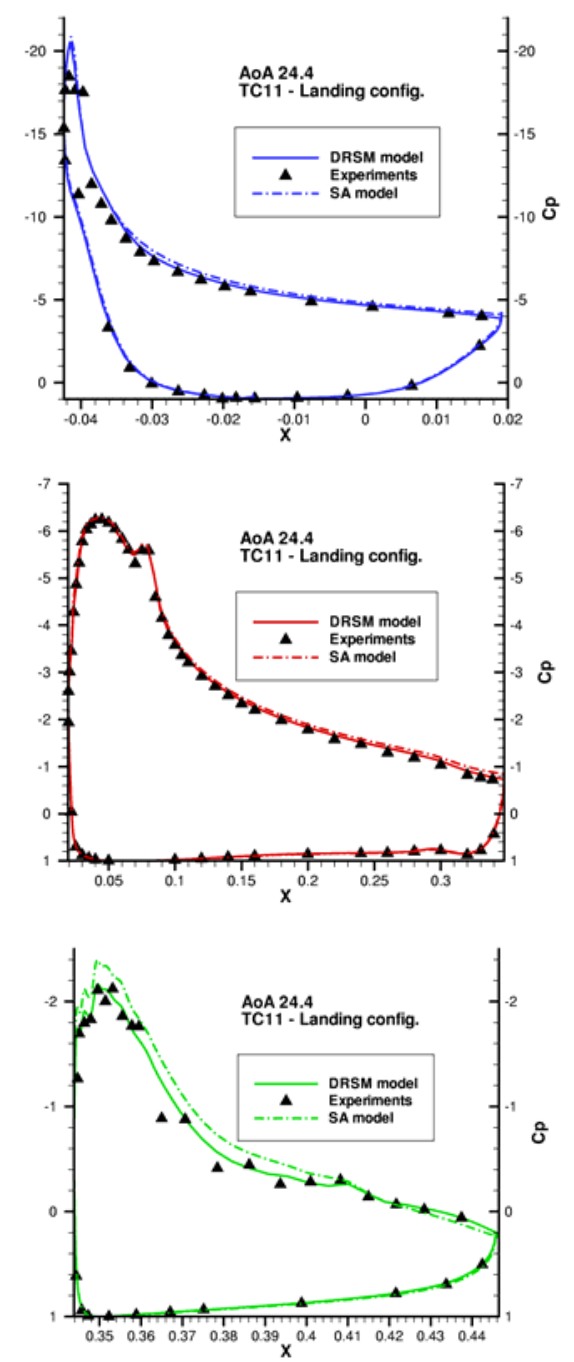

Fig. 1. Configuration TC11 of Eurolift II project: landing configuration $\left(\alpha=24.4^{\circ}\right)$. Pressure coefficient distributions on the slat (above), on the main element (middle) and on the flap (below): comparison between elsA results with RSM- $\omega$ model or Spalart-Allmaras model and experimental values.

\subsection{Aeroelasticity}

A major example of new capability implemented in elsA during the last few years is the aeroelasticity functionality [11], [12] developed by the ONERA/DDSS department (Structural Dynamics and Coupled Systems) and integrated by the ONERA/DSNA department (Computational Fluid Dynamics and Aeroacoustics). The aeroelasticity module developed in elsA currently allows the following simulations:

- harmonic forced motion simulations ;

- static coupling simulations (using either a reduced flexibility matrix or a modal approach);

- dynamic coupling simulations (using a modal approach).

As an example, we present the result of a static coupling simulation for a civil aircraft configuration at transonic regime (large winglet reference case of a wing/body/pylons/nacelles configuration in the European AWIATOR project, Mach number equal to 0.82 , lift coefficient equal to 0.49 ). The multiblock mesh associated with the half aircraft includes 93 blocks and more than 7 million points. In the CFD part of the calculation, the RANS equations with Spalart-Allmaras 
turbulence model are solved using centered space discretization, backward Euler time integration, LU-SSOR implicit technique and multigrid convergence acceleration. The computation is run in parallel mode on 8 processors starting from a flight shape estimated by simple engineering tools. Good convergence is achieved after 2000 CFD time steps: firstly 1000 pure CFD steps, and secondly 1000 CFD steps with fluid-structure coupling every 100 steps. Fig. 2 illustrates the large difference between the ground shape and the computed flight shape, and shows the good agreement between the computed results and the flight test measurements on the bending deformation and the twist angle deformation.
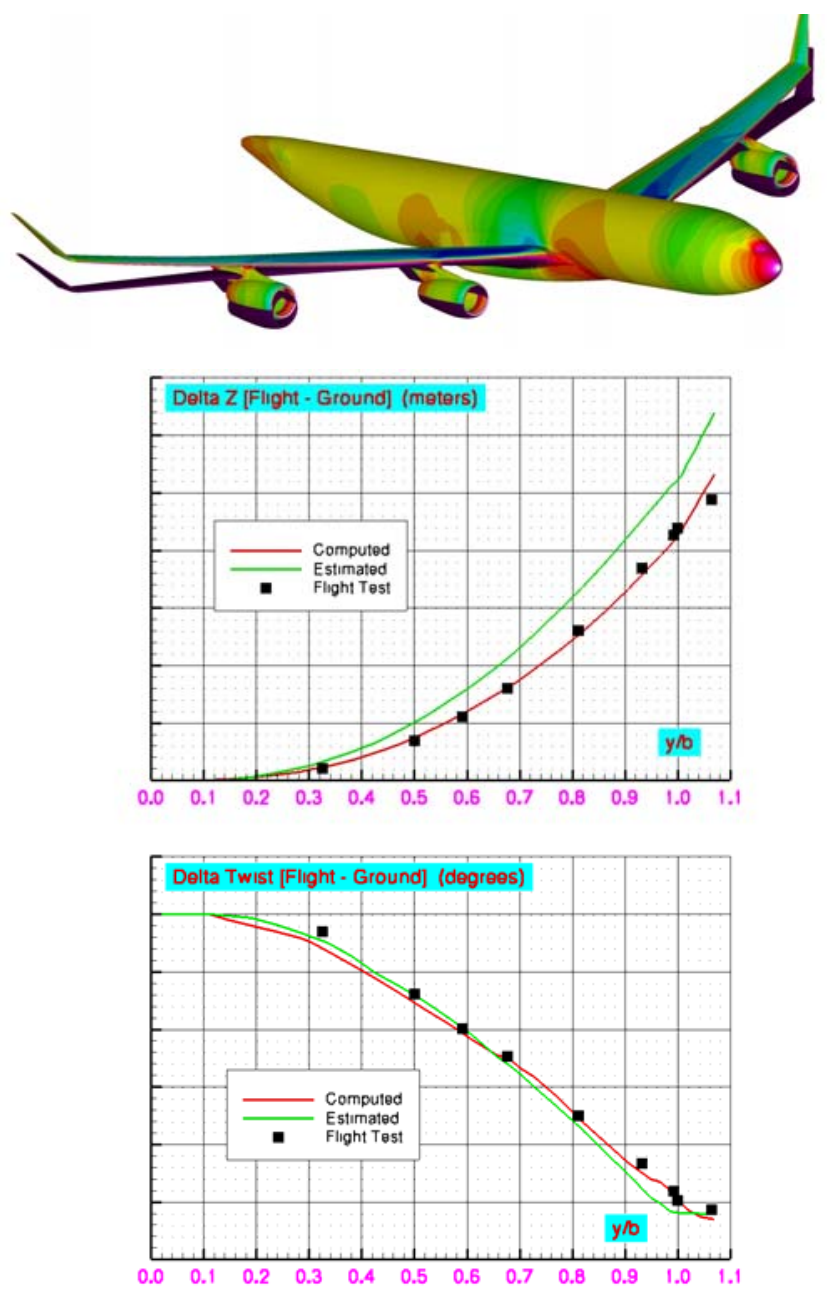

Fig. 2. Static Coupling validation with els $A$ - Difference between computed flight shape and ground shape, and computed wall pressure field (above). Spanwise evolutions of bending deformation (middle) and twist deformation (below) : comparison between initial estimation, numerical simulation results and flight test data.

The development of linearized unsteady aerodynamic functionalities is also in progress in elsA. The approach consists in solving linearized Euler or Navier-Stokes equations for structural harmonic motion. Field and mesh motion are splitted into a steady part and a first order perturbation. The approach is presently available for Euler equations and will be soon available for Navier-Stokes equations.

\section{Unstructured-hybrid RANS code TAU}

\subsection{General description}

As described in [13], [14] and [15], TAU is a CFD simulation platform which can be used from the low subsonic to the hypersonic flow regime, employing unstructured-hybrid grids composed of hexahedrons, prisms, tetrahedrons and pyramids. In TAU, the compressible 3-D Reynolds averaged Navier-Stokes equations for rigid bodies in arbitrary motion are solved by a finite-volume method with second order upwind or central space discretization with scalar or matrix artificial dissipation. The reference version of TAU uses a vertex centered dual mesh formulation. The discrete equations are integrated explicitly by multistage Runge-Kutta schemes, using local time stepping and multigrid acceleration. An implicit LU-SGS scheme is available. For time accurate computations, the implicit dual time stepping method is employed. Preconditioning is used for low speed flow simulations. Various turbulence models are available including 1 - and several 2-equation eddy viscosity models. Currently a full Differential Reynolds Stress Model is being integrated following the implementation of FLOWer ([9], [10]). Furthermore, the code offers several options for DES (see [16] in this symposium). For transition prediction on airfoils and wings, TAU is coupled to a laminar boundary layer code and an $\mathrm{e}^{\mathrm{N}}$-database method (see [17] in this symposium). The Chimera technique enhances the flexibility of TAU with respect to independently moving bodies (see [18] in this symposium). For the simulation of aeroelasticity phenomena, TAU has been extended to allow geometry and mesh deformation (see [19] and [20] in this symposium). A key feature of TAU is the grid adaptation capability for hybrid meshes based on local grid re- and derefinement and wallnormal mesh movement in semi-structured near-wall layers, allowing to efficiently resolve detailed flow features. Recently an adjoint solver has been developed within the German CFD project MEGADESIGN which allows efficient gradient-based shape optimization and goal-oriented mesh adaptation (see [21] in this symposium).

The TAU code is not a single code but is composed of a number of modules and libraries to allow easier development, maintenance and reuse of the code or parts of it. The different modules of TAU can both be used as stand-alone tools with corresponding file $\mathrm{I} / \mathrm{O}$ or within a Python scripting framework which allows also for inter-module communication without file- $\mathrm{I} / \mathrm{O}$, i.e. using common memory allocation. One of the important features of TAU is its high efficiency on parallel computers and its optimization for cache processors through specific edge colouring procedures. Parallelization is based on domain decomposition and the message passing concept using MPI.

Let us focus now on two examples of progress carried out in CFD capabilities for transport aircraft applications during the three years of the MIRACLE project.

\subsection{Convergence acceleration methods}

One objective concerning TAU has been to improve the efficiency of the existing hybrid flow solver for large scale simulations. In 2004, at the beginning of the MIRACLE 
project, most of the calculations were relying on the explicit integration of the discrete equations by multistage RungeKutta schemes, with additional convergence acceleration by a multi-grid algorithm based on agglomerated coarse grids. The development and the use of implicit methods have allowed DLR to improve the efficiency of TAU. The LU-SGS method has been selected because this method has low memory requirements, low operation counts and can be parallelized with relative ease. An example of the performance improvement achieved is given in Fig. 3 for the RANS simulation of the flow over a delta wing at a Mach number equal to 0.5 and an angle of attack of 9 degrees. Fig. 3 shows the residual and the rolling moment against iteration count. In terms of iterations, LU-SGS can be seen to converge approximately twice faster than the Runge-Kutta scheme. Moreover, one iteration of LU-SGS costs roughly $80 \%$ of one Runge-Kutta step on the Linux cluster where the calculations were performed. This results in a reduction of the overall calculation time by a factor of 2.5 .
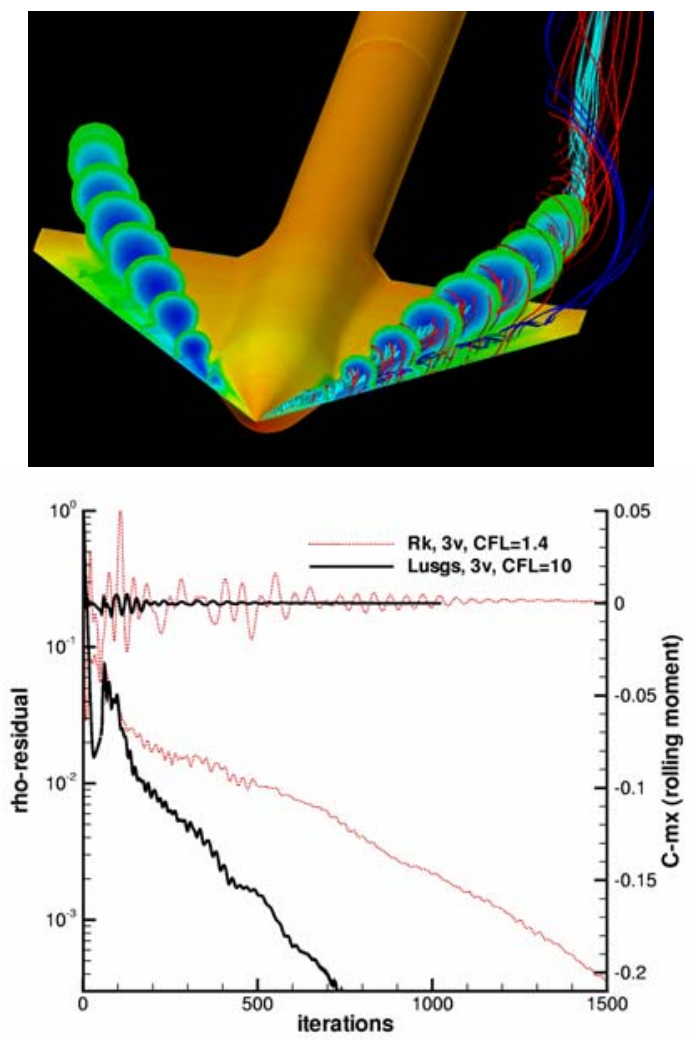

Fig. 3. Convergence improvement with implicit method in TAU, delta wing, $\mathrm{M}=0.5, \alpha=9^{0}$.

\subsection{Grid adaptation}

Local grid refinement is an essential ingredient of the hybrid flow solver TAU. It allows an accurate and efficient simulation of local phenomena by automatically adapting the grid to a given solution. At the beginning of MIRACLE project, TAU adaptation tool was supporting refinement and derefinement of tetrahedrons, prisms and pyramids. During the project, substantial progress was made by DLR on this adaptation tool. Today, the adaptation suite is fully parallelized. A dynamic parallel load balancing is available for adapted grids. The adaptation of unstructured grids with hexahedral elements is in progress. This last point is very important since Airbus has asked DLR to develop an interface between the TAU solver and the British mesh generator named SOLAR, which provides with hexahedral elements.

One example of grid adaptation for load computations of a transport aircraft with satellite antenna is shown in Fig. 4. The picture above represents the initial viscous grid, whereas the picture below shows the grid obtained after adaptation. Using local grid refinement the accuracy of load predictions has been verified.
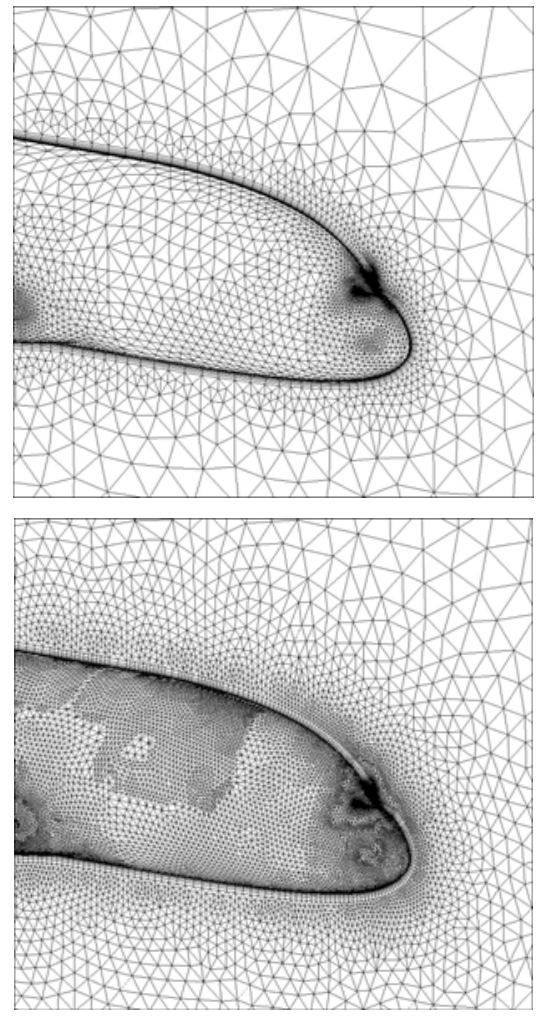

Fig. 4. Application of the TAU adaptation tool to the integration of an antenna on top of the fuselage (above : initial viscous grid; below : adapted grid).

\section{Examples of validation}

The aim of the validation activity in MIRACLE project has been to demonstrate the capability of the structured and unstructured-hybrid Navier-Stokes solvers elsA and TAU to accurately predict viscous flows for complex aircraft configurations under industrial conditions. A large spectrum of configurations was defined at the beginning of the project, including cruise configurations (AS28G wing/body configuration and DLR F6 wing/body/pylone/nacelle configuration), spoiler and aileron configurations (from HiReTT data base), high lift configurations (from the European project EUROLIFT II). MIRACLE meetings have also given opportunity to DLR and ONERA to exchange information on results obtained by elsA and TAU on other transport aircraft configurations.

\subsection{AS28G cruise configuration : ONERA calculations with elsA and TAU}

For the AS28G wing/body configuration of the GARTEUR AG39, computations were performed by the ONERA/DAAP 
department (Applied Aerodynamics) with both the elsA and the TAU software packages for a Mach number of 0.8 , an angle of attack corresponding to a lift coefficient of 0.5 and a Reynolds number of 10.4 million. Fig. 5 shows the structured and hybrid grids, used for elsA and TAU respectively.
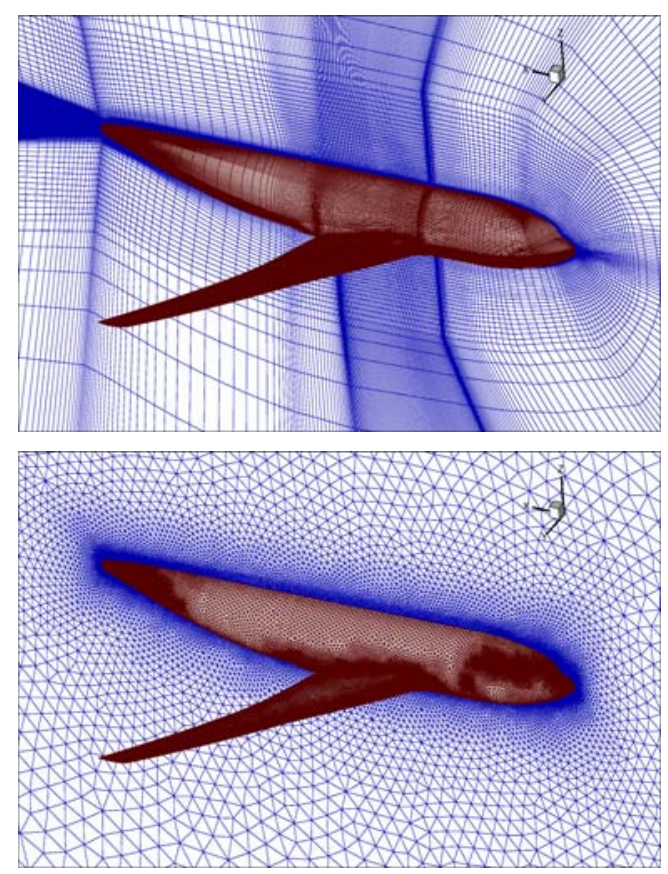

Fig. 5. AS28G Wing/Body configuration - ICEM Structured mesh; CentaurSoft Hybrid mesh.

The pressure coefficient distributions shown in Fig. 6 indicate a good match between the results from the two software packages obtained with the same turbulence model (Spalart-Allmaras model) and a satisfying comparison with the experimental values.
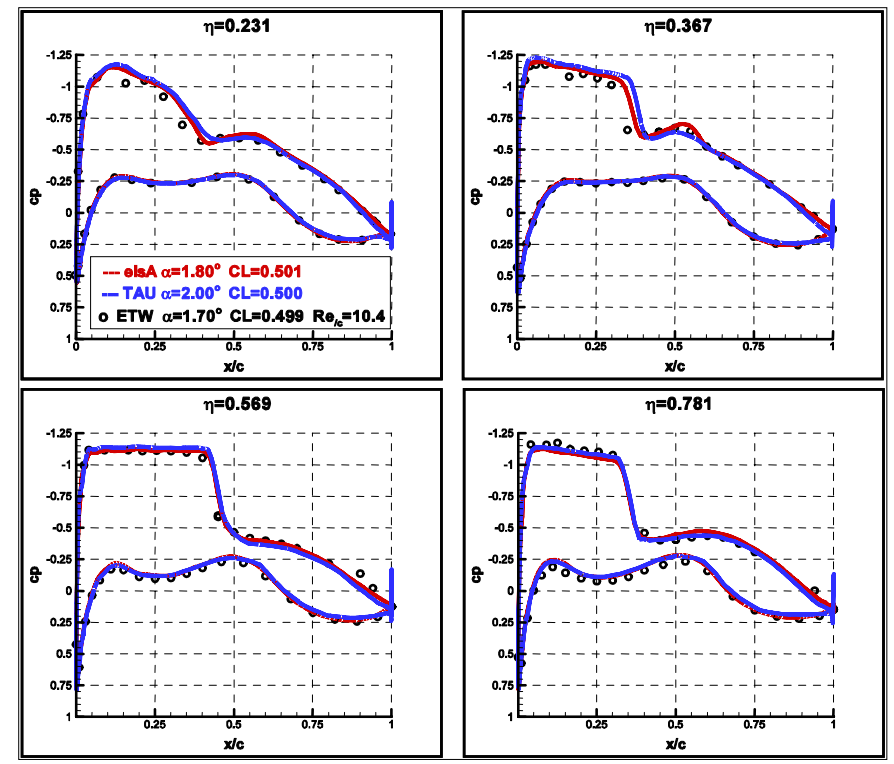

Fig. 6. AS28G Wing/Body configuration - Pressure distributions in 4 wing sections (elsA and TAU calculations performed by ONERA/DAAP).

The code-to-code comparison was pursued doing an analysis of the two solutions by the ONERA drag extraction tool (see [22] for a presentation of this tool and further details on its application to AS28G elsA and TAU calculations). As a general trend, it was found that the pressure drag calculated with TAU is higher than calculated with elsA whereas the friction drag of TAU is less than the value of elsA. A low spurious drag is obtained for both codes.

\subsection{DLR-F6 cruise configuration : DLR calculations with $T A U$}

For the F6 wing/body/pylon/nacelle configuration, computations were performed by DLR with the TAU code for a Mach number of 0.75 , an angle of attack corresponding to a lift coefficient of 0.5 and a Reynolds number of 3 million. A detailed study was done by DLR to assess the influence on the flow solution of grid density, trailing edge geometry, turbulence model and numerical artificial dissipation. As an example, Fig. 7 shows the influence on the flow topology at the extrados of the wing, of the type and the amount of numerical artificial dissipation.
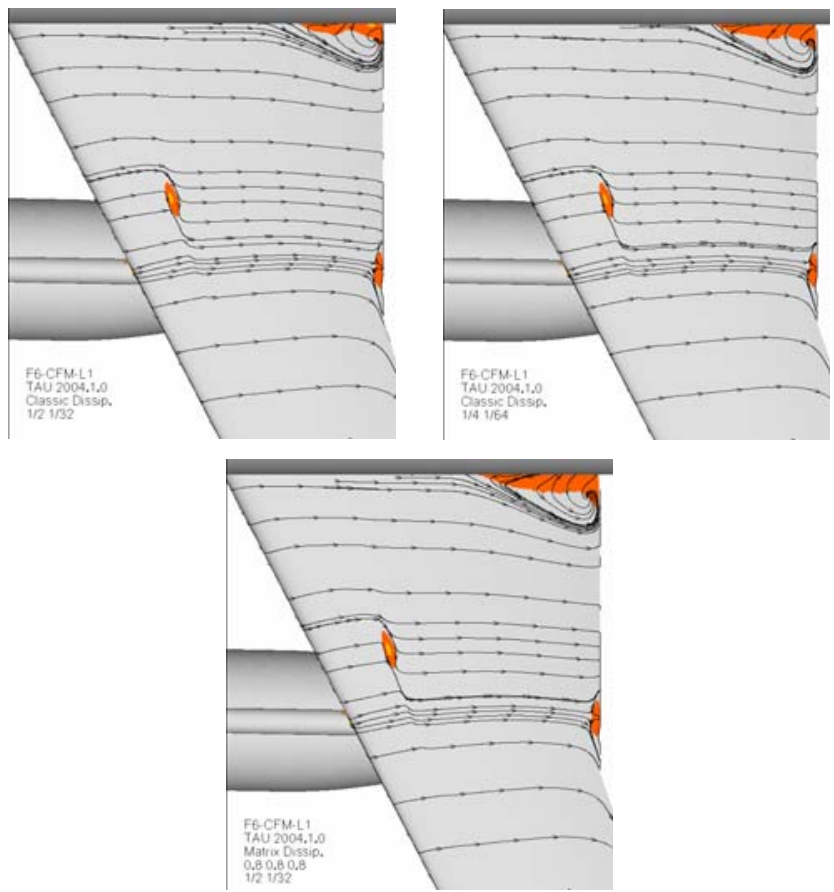

Fig. 7. Validation of TAU code on $\mathrm{F} 6$ configuration $(\mathrm{M}=0.75, \mathrm{CL}=0.5, \mathrm{Re}=3$ $\left.10^{6}\right)$ : influence of artificial dissipation.

\subsection{HiReTT aileron configuration : ONERA calculation with elsA}

A specific task within the validation exercise in MIRACLE project was the assessment of spoiler and aileron configurations based on the HiReTT data base. In particular, the effects of the aileron deflection were studied by ONERA/DAAP with elsA for a Mach number of 0.85 , an angle of attack corresponding to a lift value of 0.5 and a Reynolds number of 8.2 million. The elsA calculations are done in a 6-Million point mesh using the patch grid technique and Spalart-Allmaras turbulence model. Comparison of the results of the calculations with or without deflection of the aileron is done in Fig. 8 with the experimental values on pressure distributions in two spanwise grid sections in the aileron region. The comparison is satisfactory, if one takes into account the possible aeroelasticity effects. 


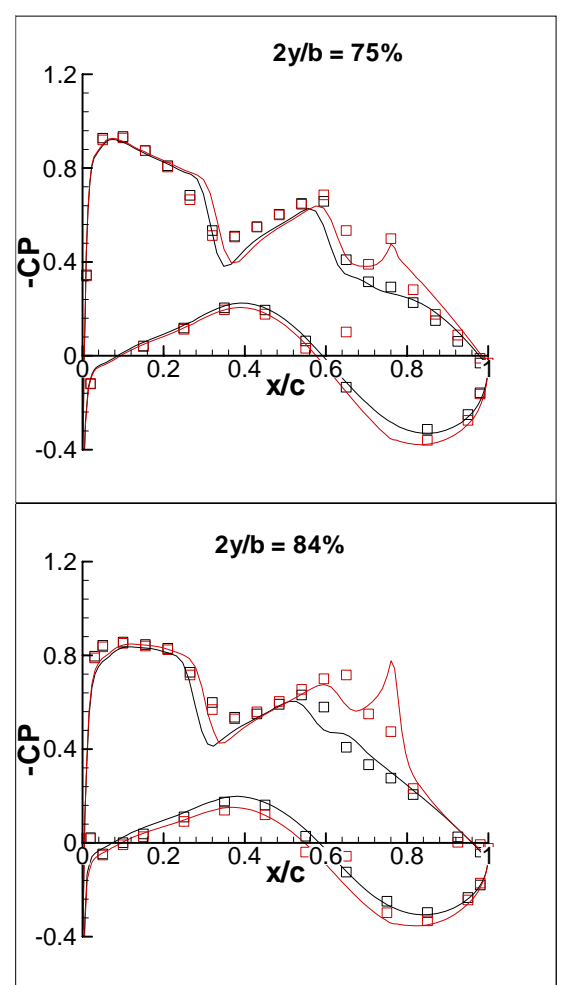

Fig. 8. Prediction with elsA of aileron deflection in HiReTT configuration (calculations : solid line, experiment : squares ; black colour : no deflection of the aileron, red colour : deflection of the aileron equal to 3 degrees).

Chimera functionality in elsA is also used for the prediction of control surface deflection effects [23, 24]. In particular, the effects of spoiler deflection have been studied by ONERA with $e l s A$ in the MIRACLE project for a wing-body + spoiler + aileron + inner wing trailing edge flap configuration in a Chimera grid provided by DLR.

\subsection{High lift configuration : ONERA calculation with elsA}

ONERA/DAAP has performed calculations with the elsA code in the framework of the EUROLIFT II project for the KH3Y high lift configuration. The multi-block mesh which was provided by Airbus Germany (Fig. 9) includes 97 blocks and 5.5 million nodes. The calculations were carried out on a HP cluster with good parallel efficiency. Mach number, angle of attack and Reynolds number are respectively equal to 0.174 , 16 degrees and 1.34 million. Spalart-Allmaras turbulence model is used. The pressure distributions shown in Fig. 9 are in good agreement with experimental data.

Other high lift calculations with $e l s A$, related to flow control, are also presented in this symposium [25].

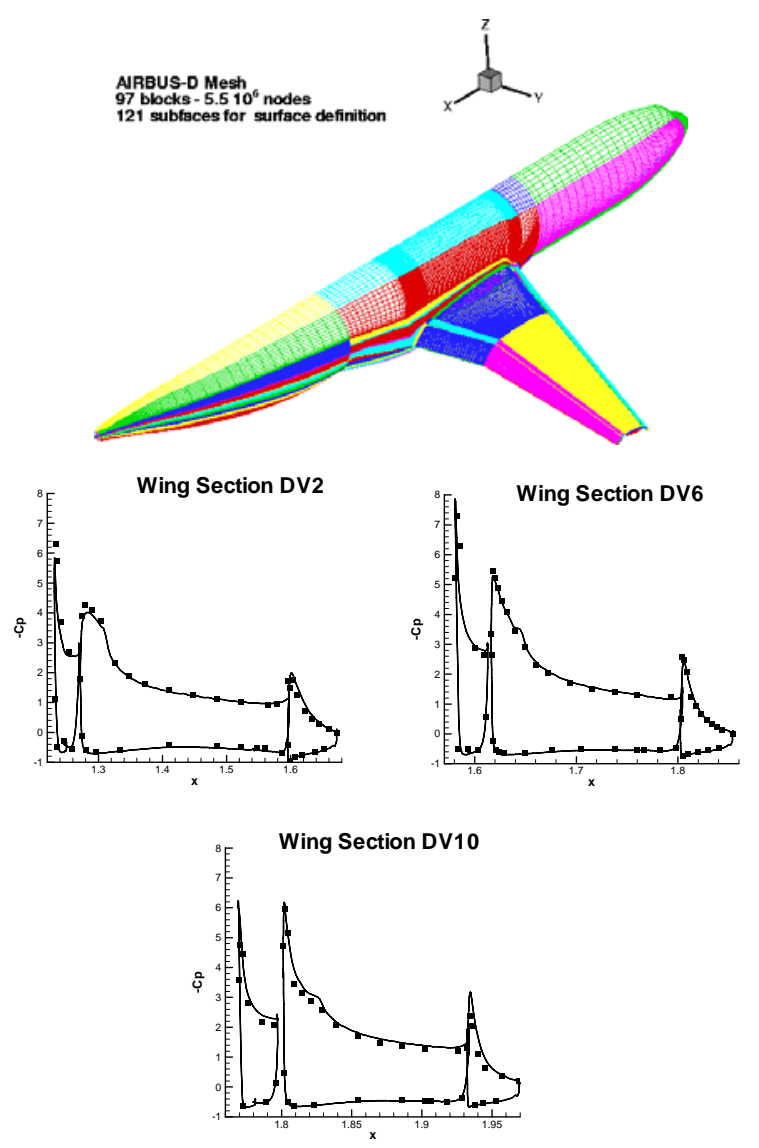

Fig. 9. elsA calculation on KH3Y high lift configuration : Mesh and pressure distributions.

\subsection{High lift configuration : DLR investigation of nacelle strakes using TAU}

For a transport aircraft high lift configuration DLR investigated the effect of nacelle strakes on maximum lift. [26]. The configuration consists of fuselage, wing, four engines and pylons, flap track fairings and deployed droop nose, slats, and flaps. The effect of grid density was investigated in detail. In a first approach a grid adaptation strategy has been used to refine the initial grid by several refinement steps using the same setting for the configurations with and without strakes. Using this approach the lift increments due to the presence of the nacelle strake could not be simulated. In a second approach the initial grid had already refined areas especially at the location of the strake vortices. Then two adaptation steps have been performed for the configuration with nacelle strakes. The grid for the configuration without strakes has been generated by filling strakes with tetrahedral elements (Fig. 10). Thus the grids for the two configurations are as similar as possible. Using this technique the increase of maximum lift due to the strakes could be computed successfully and the strake vortex dissipates significantly later. 

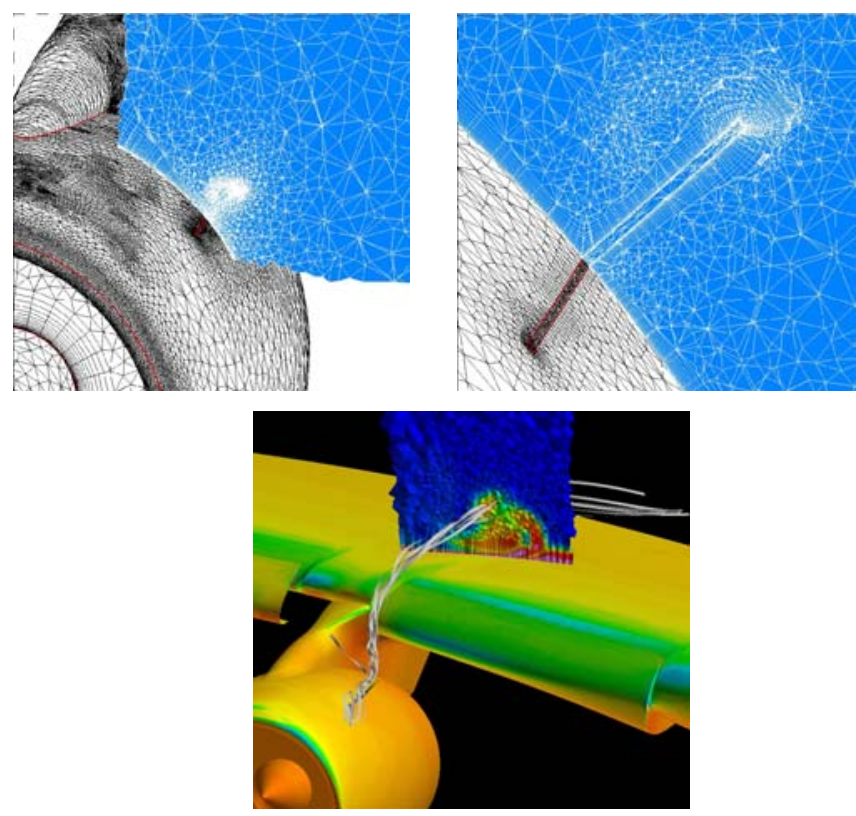

Fig. 10. Simulation of nacelle strake vortex of transport aircraft high lift configuration using TAU.

\subsection{High lift configuration : DLR coupled CFD-CSM calculation using TAU}

The DLR activities for code validation on high lift configurations are also carried out in the framework of the EUROLIFT II project. Concerning coupled CFD-CSM studies, reference data to calibrate the structural high lift wing model were provided in a DLR static deformation test of the KH3Y model without wind. A coupled CFD-CSM computation for the complex high lift configuration under ETW flow conditions was carried out featuring a coupling of the TAU code and a structural ANSYS FEM model [20]. Fig. 11 shows the computed deformation of the KH3Y configuration.

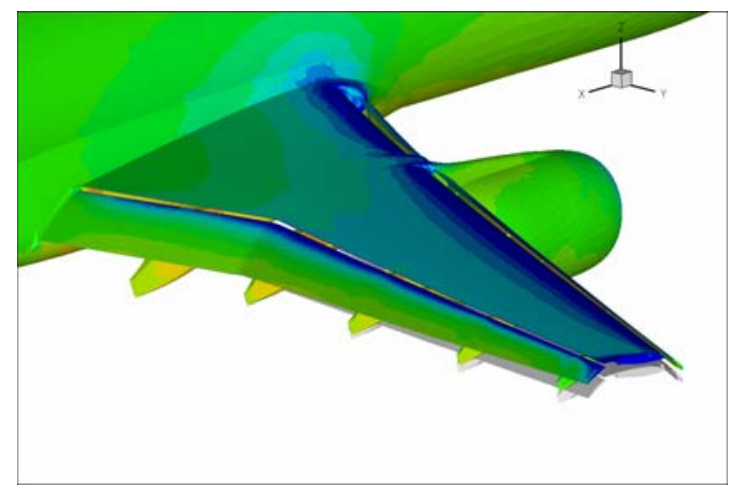

Fig. 11. Pressure distribution on KH3Y landing configuration, coupled TAU/ANSYS computation, $\mathrm{M}=0.2, \alpha=11.2^{\circ}$.

\subsection{Off-design configuration : ONERA calculation of the buffet phenomenon with elsA}

ONERA/DAAP has performed DES simulations with elsA around the OAT15A airfoil. The computation result shown here has been obtained with a Zonal - DES approach applied for a free-stream flow and with an infinite wing hypothesis. Flow conditions are the following:
$\mathrm{M} \infty=0.73, \mathrm{Pi}=10^{5} \mathrm{~Pa}, \mathrm{Ti}=300 \mathrm{~K}, \mathrm{Rec}_{\mathrm{c}}=3.10^{6}$ and the angle of attack is equal to $3.5^{\circ}$. For these flow conditions, the buffet phenomenon is characterized by a wide movement of the shock, caused by the separation. As presented in Fig. 12, the Zonal - DES approach allows turbulent flow structures to be calculated in the separated area region. This accurate simulation is possible thanks to accurate time and space resolution with adapted meshes (see [5] in this symposium for more details on this calculation).

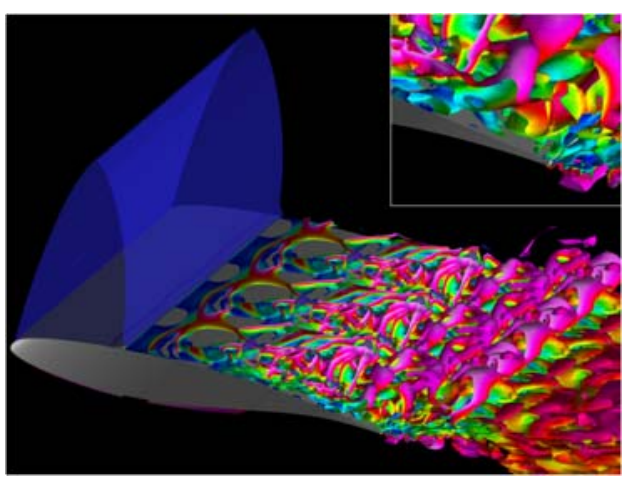

Fig. 12. Simulation of the buffet phenomenon with els $A-$ surface blue $=$ iso surface Mach number equals 1 - coloured surfaces = iso-Q criterion surfaces $\alpha=3.5^{\circ}$.

\section{Advanced software architecture}

\subsection{General context}

Work done by DLR and ONERA on this topic corresponds to activities preparing the design of a future advanced software architecture which should offer the flexibility for the integration of classical structured and unstructured-hybrid finite volume methods within one single toolset and the extension for future innovative CFD-technologies. During the first part of MIRACLE project, DLR and ONERA studied the feasibility of a short-term development of a complete advanced architecture mixing structured and unstructuredhybrid methods, starting from the TAU code in combination with the Object-Oriented know-how of ONERA and the elsA code. Yet, time and manpower were not sufficient in MIRACLE project to achieve next generation software in MIRACLE timescale, especially after the failure of the OPERA project. This project which was submitted within the 2nd Call of the European 6th framework would have dealt with the development of a European advanced CFD kernel which would probably have been based on elsA and TAU. The focus has then been shifted from the short-term complete advanced architecture based on fine grain components, to the definition of a simulation process based on easy wrapping techniques, such as Python, to integrate medium or large grain code components as a set of services.

\subsection{Results of DLR/ONERA work on advanced software architecture}

In this initial study carried out during the first year of MIRACLE project, DLR worked on the demonstration of the prototype of an hybrid code with incorporated structured 
algorithms, whereas ONERA and Cerfacs (which is partner of ONERA in the development of elsA) worked on an ObjectOriented (OO) redesign of parts of TAU kernel and on the reuse of elsA design elements inside TAU.

Work of DLR led to a prototype named TAUijk which exhibited promising results in comparison with the blockstructured code FLOWer [27]. DLR now plans to transfer the techniques developed in the prototype into the central version of the TAU-code. However, since TAUijk is based on a cellcentered metric, this cell-centered formulation has first to be included in TAU before transferring the techniques from TAUijk to TAU. The extension of TAU to the cell-centered metric is almost finished.

Work of ONERA and Cerfacs provided DLR with information on how TAU kernel could evolve toward an OO redesign. Thus, it prepared a better future elsA-TAU software consistency, even if ONERA and Cerfacs developments have not directly been integrated in the central TAU version. Today, software technical choices between elsA and TAU are closer than they were at the beginning of MIRACLE project. In particular, some new developments in TAU are today directly written in $\mathrm{C}++$, which is the main programming language in elsA. Besides, Python scripting language is used in both DLR and ONERA as an easy wrapping technique.

\subsection{Integration of large grain components in the Airbus simulation process}

In order to focus on the integration of large grain components in a simulation process, DLR and ONERA contributed to the design of the Airbus FlowSimulator architecture. It consists of a data manager (FSDM), which allows for import and export of data via a Python interface. All components providing services to the flow simulator environment have to contain an interface to the FSDM and a Python layer which allows for steering of the component.

Within TAU, Python interfaces were designed and implemented in the so-called TAU-PY interface for control of TAU functionalities from a top level steering script. For the exchange of data between TAU modules, a data management was set up which transfers the data directly between the modules (not via Python) hidden for the user and thus for the Python scripting. For the integration of TAU into the FlowSimulator environment an interface to the TAU data manager (TAU-DM) was built which allows for import and export between TAU-DM and Python. A set of interfaces between TAU-PY and FSDM is progressively built, and completed stepwise according to the availability of interfaces in FSDM for unstructured data appropriate to TAU.

In elsA, a strategy of interoperability between components was developed, based first on the adoption of the CGNS standard as a common data structure and second on an inmemory exchange mechanism. A Python interface fully CGNS compliant was implemented in the purpose to use the elsA solver as a CGNS component. Within this interface, an elsA computation can be described in a CGNS logical tree structure usable by any CGNS component. This strategy was chosen to integrate els $A$ into the FlowSimulator architecture, considering CGNS as the basis for all in-memory exchanges to or from the FSDM, as for all to or from the elsA solver (as it was already done previously for helicopter blade fluid-structure strong coupling [28]). Specification and development of the elsACGNS interface allow the definition and progressive implementation of a similar Python FSDM-CGNS interface.

\section{Conclusion}

The joint effort of DLR and ONERA on CFD development has accelerated the enhancement of numerical methods for aerodynamic predictions within conventional and unconventional, environmentally friendly aircraft designs. Both, flow solvers elsA and TAU are routinely used in European aircraft industry. The MIRACLE effort is an important step on the road map to meet the future ambitious goals of aircraft research and industry.

Among the most significant achievements of the MIRACLE co-operation, we can mention:

- the fruitful exchanges on CFD topics, such as convergence acceleration methods, Chimera grid techniques, turbulence and transition modelling;

- activities involved in cross-validating the two software packages, els $A$ and TAU, including use of drag extraction tools developed by the ONERA/DAAP department for the analysis of the solutions obtained by the two packages;

- the fruitful exchanges on quality policy and software management of elsA and TAU (integration, versioning and validation processes, documentation, support ...);

- work covering the search for better coherence of software architecture of the two software packages.

The six meetings of the MIRACLE project have also been an opportunity for ONERA and DLR to exchange information on other projects they are conducting in CFD and even to harmonise their participation, as was the case for the European NEFA and ROSAS projects and for the GARTEUR group on the AS28G wing/fuselage configuration.

The relationships built up between DLR and ONERA in CFD for transport aircraft during the MIRACLE project should enable them to continue with, or even intensify, the cooperation in CFD in the coming years.

Firstly, DLR and ONERA will continue the development of components which should be in favour of an exchange of components in the future. Let us stress that the distinction between types of meshes today considered by elsA and TAU is not so important as it could first appear, for the two following reasons. First reason is that there are quite a lot of functionalities which are or could be independent of the types of CFD meshes. In particular, the strong development of multidisciplinary activities starting from aerodynamics (aeroacoustics, aerothermics, aeroelasticity ...) provides such functionalities. Second reason is that elsA and TAU will progressively enlarge the types of CFD meshes they consider. DLR has already begun to develop in TAU a specific solver for structured meshes, called TAU-IJK and will carry-on this development. ONERA has recently begun the development of an unstructured capability in elsA. Work on Cartesian background meshes is also planned in particular for helicopter applications in the two softwares. If one considers the development plans of TAU and elsA in the next few years, these two software families have still a long life, and the interest of both DLR and ONERA is that each software family takes advantage of the other software family through co- 
operative activities.

Secondly, a new co-operative project on CFD is presently in preparation by DLR and ONERA. This project, called IMPULSE (Innovative Methods for Prediction of Unsteady Aerodynamics Data for Loads, Stability and Control for Complete Flight Envelope) should last from 2007 to 2010 and will address the objective of developing advanced CFD tools for high-fidelity numerical simulations in early stages of aircraft design. More precisely, the expected outputs should allow virtual flight-tests (by numerical simulations) for selected critical points of the flight envelope by coupling of aerodynamics, flight mechanics, flight control and structure in a common time loop. Several institutes and branches/departments from DLR and ONERA will be involved in this project.

\section{References}

[1] K. Pahlke, M. Costes, A. d'Alascio, C. Castellin and A. Altmikus, The French-German Helicopter-CFD-Project CHANCE, ODAS symposium, Toulouse, Oct. 2006.

[2] L. Cambier and M. Gazaix, elsA: an efficient ObjectOriented solution to CFD complexity, 40 $0^{\text {th }}$ AIAA Aerospace Science Meeting and Exhibit, Reno, Jan. 2002.

[3] M. Gazaix, A. Jollès and M. Lazareff, The elsA ObjectOriented tool for industrial applications, 23rd ICAS meeting, Toronto, Sept. 2002.

[4] C. Benoit, G. Jeanfaivre and E. Canonne, Synthesis of ONERA Chimera method developed in the frame of CHANCE program, 31st European Rotorcraft Forum, Florence, Sept. 2005.

[5] V. Brunet, S. Deck, L. Jacquin and P. Molton, Transonic Buffet Investigation using Experimental and DES techniques, ODAS symposium, Toulouse, Oct. 2006.

[6] J. Cliquet and R. Houdeville, New developments in transition computation in the elsA Navier-Stokes solver, ODAS symposium, Toulouse, Oct. 2006.

[7] J. Peter, Discrete Adjoint Method in elsA : Theory, ODAS symposium, Toulouse, Oct. 2006.

[8] G. Carrier, S. Mouton and I. Salah El Din, Discrete Adjoint Method in elsA : Application to Aerodynamic Design Optimisation, ODAS symposium, Toulouse, Oct. 2006.

[9] B Eisfeld, and O. Brodersen, Advanced Turbulence Modelling and Stress Analysis for the DLR-F6 Configuration, AIAA-Paper 2005-4727, 2005.

[10] B. Eisfeld, Computation of Complex Compressible Aerodynamic Flows with a Reynolds Stress Model, Conference on Boundary and Interior Layers, BAIL 2006, Göttingen, Germany, July 2006.

[11] $\mathrm{Ph}$. Girodroux-Lavigne, Progress in steady/unsteady fluidstructure coupling with Navier-Stokes equations, IFASD 2005, Munich, Germany, June 2005.

[12] A. Dugeai, Aeroelastic developments in the elsA code and unsteady RANS applications, IFASD 2005, Munich, Germany, June 2005.

[13] T. Gerhold, M. Galle, O. Friedrich and J. Evans, Calculation of Complex 3D Configurations Employing the DLR TAU-Code, AIAA paper 97-0167, 1997.

[14] D. Schwamborn, Th, Gerhold and R. Heinrich, The DLR TAU-code: recent applications in research and industry, ECCOMAS CFD 2006 meeting, Delft, Netherlands, Sept. 2006.

[15] N. Kroll, K. Becker, H. Rieger and F. Thiele, Ongoing activities in flow simulation and shape optimization within the German Megadesign project, 25 $5^{\text {th }}$ ICAS meeting, Hamburg, Sept. 2006.
[16] H. Lüdeke, Detached Eddy Simulation of a Delta Wing With Rounded Leading Edge, ODAS Symposium, Toulouse, Oct. 2006.

[17] A. Krumbein, $\mathrm{e}^{\mathrm{N}}$ transition prediction for 3D wing configurations using database methods and a local, linear stability code, ODAS symposium, Toulouse, Oct. 2006.

[18] A. Schütte, G. Einarsson, A. Raichle, B. Schöning, Th. Alrutz, J. Neumann, J. Arnold, W. Mönnich and T. Forkert, Simulation of Maneuvering Aircraft, ODAS Symposium, Toulouse, Oct 2006.

[19] T. Gerhold, Efficient Algorithms for Mesh Deformation, ODAS Symposium, Toulouse, Oct. 2006.

[20] R. Heinrich, J. Wild, Th. Streit and B. Nagel, Steady Fluid Structure Coupling for Transport Aircraft, ODAS Symposium, Toulouse, Oct. 2006.

[21] R. Dwight, J. Brézillon and D. Vollmer, Efficient Algorithms for Solution of the Adjoint Compressible Navier-Stokes Equations with Applications, ODAS Symposium, Toulouse, Oct. 2006.

[22] S. Esquieu, M. Meheut and H.F. von Geyr, Far-Field Drag Extraction from Numerical Solutions and Wake Surveys, ODAS symposium, Toulouse, Oct. 2006.

[23] G. Fillola, M.-C. Le Pape and M. Montagnac, Numerical simulations around wing control surfaces, $24^{\text {th }}$ ICAS meeting, Yokohama, Sept. 2004.

[24] G. Fillola, G. Carrier, A.-M. Rodde and J.-B. Dor, Experimental study and numerical validation of flows around wing control surfaces, $25^{\text {th }}$ ICAS meeting, Hamburg, Sept. 2006.

[25] M. Meunier and V. Brunet, High-Lift Devices Performance Enhancement Using Flow Control Strategies, ODAS Symposium, Toulouse, Oct. 2006.

[26] O. Brodersen and J. Wild, DLR-IB 124-2004-18, 2004.

[27] R. Heinrich and A. Schwöppe, Implementierung und Nutzung strukturierter Algorithmen im unstrukturierten TAU-Code, STAB Symposium, Darmstadt Germany, Nov. 2006.

[28] M. Poinot, M. Costes and B. Cantaloube, Application of CGNS software components for helicopter blade fluidstructure strong coupling, $31^{\text {st }}$ European Rotorcraft Forum, Florence, Sept. 2005. 